\title{
Perioperative Cryoprecipitate Waste Reduction
}

\author{
Camden Burk $^{1} \cdot$ Kent Eliason $^{2} \cdot$ Allison Doney $^{1} \cdot$ Jeremi Mountjoy $^{1} \cdot$ Adam A. Dalia $^{1}$ (D)
}

Received: 7 December 2020 / Accepted: 1 February 2021 / Published online: 10 February 2021

(C) The Author(s), under exclusive licence to Springer Science+Business Media, LLC part of Springer Nature 2021

\begin{abstract}
Improvement of operating room efficiency regarding perioperative blood product transfusion is fundamental for surgical patients. The aim of this study was to 1) assess the use of cryoprecipitate in the operating room at our institution 2) identify and address gaps in knowledge regarding the process of ordering and returning cryoprecipitate 3) aim to reduce cryoprecipitate wastage by 50\% over a 14-month period. Institutional data from Dec. 2017 to April 2020 was retrieved on cryoprecipitate that was ordered, thawed, and delivered to the operating room. Additionally, data was collected regarding cryoprecipitate that was wasted. A retrospective analysis of weekly intraoperative cryoprecipitate utilization was performed to compare wastage before and after implementation of interventions. Pre-intervention (Dec 2017 - March 2019), a total of 453 units of cryoprecipitate were issued with 14 units wasted (3\% wastage). Between March 2019 and April 2020, the 14 months after our intervention, there were 402 units of cryoprecipitate issued with only 1 unit wasted ( $0.25 \%$ wastage). The overall cryoprecipitate waste rate was reduced by $91.66 \%$. Month-to-month comparison of pre-intervention and post-intervention data identified significant reduction in average monthly wastage $(0.875 \mathrm{vs} 0.071$ units respectively, $p<0.05)$. Appropriately, there was not a significant change in the mean monthly cryoprecipitate issued to the operating room ( 28.31 vs. 28.7 units, $p=0.94)$. These results demonstrate that utilizing educational initiatives for optimization of blood product management can reduce unnecessary ordering, transfusions, and wastage; an outcome that could ultimately reduce costs.
\end{abstract}

Keywords Operating Room efficiency · Blood transfusion · Waste Reduction · Quality improvement

\section{Introduction}

The rate of blood product use per capita in the United States is higher compared to many other industrialized countries, leading to concerns regarding the sustainability of the United States blood system [1, 2]. In recent years, guidelines regarding transfusion thresholds, prophylactic use, and storage conditions have been developed at a national and institutional level to optimize clinical practice. While there has been a steady decrease of transfusion rates for most blood

This article is part of the Topical Collection on Systems-Level Quality Improvement

Adam A. Dalia

aadalia@mgh.harvard.edu

1 Department of Anesthesia, Critical Care, and Pain Medicine, Massachusetts General Hospital, Harvard Medical School, 55 Fruit Street, Boston, MA 02114, USA

2 Department of Pathology, Blood Transfusion Medicine, Massachusetts General Hospital, Boston, MA, USA components with these guidelines, the transfusion of cryoprecipitate in the United States has increased [2-4].

Cryoprecipitate is a fraction of plasma that precipitates when fresh frozen plasma is thawed and centrifuged to remove the supernatant [5]. The remaining insoluble precipitate is enriched with clotting factors, including Factors I (fibrinogen), VIII, XIII and von Willebrand factor (vWF) [3]. Practice guidelines from the American Society of Anesthesiologists (ASA) recommends cryoprecipitate transfusion in the following circumstances: fibrinogen $<100 \mathrm{mg} /$ $\mathrm{dL}$ with microvascular bleeding, to correct excessive microvascular bleeding in massively transfused patients when fibrinogen concentrations cannot be measured in a timely fashion, bleeding with von Willebrand Disease, congenital fibrinogen deficiency [3, 6-8]. Treatment of Factor VIII deficiency, Factor XIII deficiency, and vWF deficiency with cryoprecipitate has markedly decreased due to the availability of coagulation factor concentrates. As such, in the operating room cryoprecipitate is largely used for acquired hypofibrinogenemia in the context of hemorrhage such as seen in liver transplantation, cardiovascular surgery and obstetric surgery $[5,9,10]$. 
Guidelines for cryoprecipitate are based on clinical consensus and can vary between providers and institutions due to the lack of evidence from randomized controlled trials [5, 11-13]. With this in mind, it is not a surprise that the clinical indications for the use of cryoprecipitate have changed frequently over the last few decades. These changes in guidance are likely due to a better understanding of the role of fibrinogen in processes such as coagulopathy, hemostasis, pathogen transfer, and role of alternative therapies [3, 5].

The aim of this quality improvement study was to 1) assess the use of cryoprecipitate in the operating room at our institution 2) identify and address gaps in knowledge regarding the process of ordering and returning cryoprecipitate 3) aim to reduce cryoprecipitate wastage by $50 \%$ over a 14 -month period.

\section{Methods}

This project was undertaken as a quality improvement initiative at our institution, a quaternary referral academic medical center. Institutional data was retrieved on cryoprecipitate considered "issued"; institutionally defined as cryoprecipitate that is ordered, thawed, and delivered to the operating room. Additionally, data was collected regarding cryoprecipitate that was "wasted"; defined as delivered but not transfused, not reallocating to another patient, and subsequently disposed of. At our institution once the cryoprecipitate is thawed it must be transfused within $4 \mathrm{~h}$. If the product is delivered to the operating room but is not used, it can be returned to the blood bank and reallocated to another patient if it has been less than $4 \mathrm{~h}$ since thawing and it was not returned inside of a cooler. The data was collected from the operating rooms on a weekly basis from December 2017 to April 2020.

In addition to data collection, a survey was administered to all anesthesia clinicians asking questions related to why and how cryoprecipitate was ordered, transfused, and returned (Fig. 1). The goal of this survey was to identify gaps in knowledge regarding the appropriate use of cryoprecipitate and the ordering/handling logistics which could be addressed with focused educational interventions. Three educational interventions were performed: a multidisciplinary department wide grand round session which included a blood bank representative (pathologist), an online cryoprecipitate resource platform was created reinforcing the Practice guidelines from the American Society of Anesthesiologists (Figs. 2 and 3), and education of ancillary surgical staff (anesthesia techs, operating room nurses, and operating room assistants) in the proper handling of cryoprecipitate. Data continued to be collected on a weekly basis after these interventions, from March 2019 to April 2020. A retrospective analysis of intraoperative cryoprecipitate utilization was performed before and after the implementation of these interventions. There was no consequence or penalty for ordering cryoprecipitate or wasting the cryoprecipitate unit. The collected data was anonymous and could not be linked back to a specific clinician or operating room; additionally, clinicians were not specifically notified that waste was being tracked to avoid bias in ordering/ transfusion habits.

\section{Results}

Between December 2017 and April 2020, a total of 855 units of cryoprecipitate were issued to the operating room and 15 units were wasted (Fig. 4). Pre-intervention (Dec 2017 March 2019), a total of 453 units of cryoprecipitate were issued with 14 units wasted (3\% wastage). Between March 2019 and April 2020, the 14 months after our intervention, there were 402 units of cryoprecipitate issued with only 1 unit wasted $(0.25 \%$ wastage). The overall cryoprecipitate waste rate was reduced by $91.66 \%$ (Fig. 5). Month-to-month comparison of pre-intervention and post-intervention data identified significant reduction in average monthly wastage ( 0.875 vs 0.071 units respectively, $p<0.05$ ). Appropriately, there was not a significant change in the mean monthly cryoprecipitate issued to the operating room (28.31 vs. 28.7 units, $p=0.94$ ) (Fig. 6).

\section{Statistical analysis}

The analysis included 62 weeks of pre-intervention data and 56 weeks of post-intervention data. Descriptive statistics and unpaired, 2 tailed t-tests were calculated using Microsoft Excel (Redmond, WA) to compare the pre-intervention and post-intervention time periods. A $p$ value $<0.05$ was considered significant. Of note, data was analyzed using month to month comparison.

\section{Discussion}

Improving blood product utilization, administration, and maintenance was the primary driver for this quality improvement project which focused on educational initiatives. The focused educational initiatives included: a multidisciplinary grand round, online resources for the anesthesia department, and education of operating room ancillary staff. These measures were used to address the deficiencies noted from the clinician survey to successfully reduce wastage. Our analysis of cryoprecipitate utilization revealed less cryoprecipitate was being wasted after 


\section{Cryoprecipitate Waste Survey}

What is your role in the operating room?

1. Faculty

2. Fellow

3. Resident

4. CRNA

5. SRNA

How confident is your understanding of the process of ordering cryoprecipitate from the blood bank?

1. Extremely confident

2. Very confident

3. Somewhat confident

4. Neutral

5. Not so confident

6. Not at all confident

Have you ordered cryoprecipitate in the last 3 months?

1. Yes

2. No

If you ordered cryoprecipitate in the last 3 months, for which indication?

1. Hypofibrinogenemia (fibrinogen $<100 \mathrm{mg} / \mathrm{dL}$ )

2. Inherited disorders of fibrinogen

3. Suspected disseminated intravascular coagulation (DIC)

4. Suspected uremic bleeding

5. Liver dysfunction

How long is the average wait time for cryoprecipitate to be delivered to the OR once ordered?

1. 10-20 minutes

2. 30-40 minutes

3. $60-70$ minutes

4. Over 90 minutes

If you do not end up using the cryoprecipitate how should it be returned to the blood bank?

1. Leave in the OR, outside of the blood cooler

2. Leave in the OR, inside of the blood cooler

3. Give it to the circulating nurse for return

4. Give it to the OR control desk

5. Leave it in the dumbwaiter to be sent back to the blood bank

Fig. 1 Cryoprecipitate Utilization Survey

implementation of the educational interventions. Further, the lack of statistical significance between the number of ordered cryoprecipitate units pre and post intervention signal that the reduction in waste was not solely due to an absolute reduction in total number of units ordered but a combination of more appropriate ordering and returning of cryoprecipitate (Fig. 6). The percentage of cryoprecipitate waste was effectively reduced by over $90 \%$, from a pre-intervention waste rate of $3 \%$ to a post intervention waste rate of $0.25 \%$. 
Figure 2. Departmental Online Educational Content

When should I order cryoprecipitate?

When the decision has been made that the patient requires a unit of cryoprecipitate

What are the indications for cryoprecipitate transfusion?

Should attempt to assess fibrinogen levels before the administration of cryoprecipitate, if possible. According to the American Society of Anesthesiologists (ASA) guidelines, cryoprecipitate is indicated in the following clinical scenarios:

- When a test of fibrinogen activity indicates a fibrinolysis

- When the fibrinogen concentration is less than $100 \mathrm{mg} / \mathrm{dL}$ in the presence of excessive bleeding

- As an adjunct in massively transfused patients if fibrinogen level cannot be measured or awaiting the measurement is detrimental to patient care

- Patients with congenital fibrinogen deficiencies

- Patients who are bleeding and are diagnosed von Willebrand disease if desmopressin or VWF/Factor concentrates are not available

- Inherited disorders of fibrinogen

Additional indications:

Disseminated Intravascular Coagulopathy (DIC) by clinical evaluation and as defined by International Society of Thrombosis and Haemostasis ${ }^{1}$ scoring ( 5 or higher):

- Platelet count ( $>100 \mathrm{k}=0,<100 \mathrm{k}=1,<50 \mathrm{k}=2)$

- Fibrin degradation products such as D-Dimer (no increase $=0$, moderate increase $=2$, strong increase $=3$ )

- Prolonged prothrombin time $(<3 \mathrm{sec}=0,>3 \mathrm{sec}=1,>6 \mathrm{sec}=2)$

- Fibrinogen level $(>1.0 \mathrm{~g} / \mathrm{L}=0 ;<1.0 \mathrm{~g} / \mathrm{L}=1)$

Uremic Bleeding, patients with uremia who have not responded to desmopressin

Patients with severe liver disease who are experiencing bleeding, but not for prophylactic use in low bleeding risk surgeries.

As part of a massive transfusion protocol (MTP), specifics on MTP can be found here: http://handbook.partners.org/content/pdf/MGHTransfusionMassiveTransfusionTrauma.pdf

How much volume is in a unit of cryoprecipitate?

A unit or "pool" of cryoprecipitate is between $150-200 \mathrm{~mL}$.

How long does it take to receive cryoprecipitate once ordered?

The average time is $30-40$ minutes, this may depend on the time of day and needs across the operating room. The cryoprecipitate must thaw prior to leaving the blood bank.

\section{Should I thaw a unit of cryoprecipitate just in case I need it?}

No. If you thaw a unit of cryoprecipitate it needs to be transfused within 4 hours otherwise the unit will be wasted.

Does Cryoprecipitate need to be transfused through a blood warmer?

No, the unit is room temperature and does not need to be administered through a blood warmer

Does cryoprecipitate need to be transfused through a blood filter?

Yes, a standard blood filter can be used or transfusion.

What happens if I ordered a unit of cryoprecipitate to the operating room but then I don't need it?

You can return the unit to the blood bank within 4 hours of the unit being thawed and they will attempt to re-allocate that unit to another patient.

\section{How do I return a unit of cryoprecipitate back to the blood bank?}

It should be returned to the blood bank in the plastic bag it was delivered in and should never be placed inside of the blood cooler.

Additional guidelines and FAQs can be found here:

https://ether.mgh.harvard.edu/wiki/index.php?title=Blood Bank FAQ

\section{Full ASA guidelines on blood transfusions can be found here:}

Practice guidelines for perioperative blood management. Anesthesiology. 2015;122(2):241-275.

https://pubmed.ncbi.nlm.nih.gov/25545654/

1. Toh CH, Hoots WK, SSC on Disseminated Intravascular Coagulation of the ISTH. The scoring system of the scientific and standardisation committee on disseminated intravascular coagulation of the international society on thrombosis and haemostasis: a 5-year overview. J Thromb Haemost. 2007;5(3):604-606

Fig. 2 Departmental Online Educational Content 
While the appropriate use of cryoprecipitate in the operating room is limited, common uses at our institution based on the survey included hypofibrinogenemia [defined as fibrinogen $<100 \mathrm{mg} / \mathrm{dL}$ ] ( $64.7 \%$ of responders), suspected disseminated intravascular coagulation [ $[17.7 \%$ of responders), and inherited disorders of fibrinogen (17.6\% of responders). Emergence of evidence-based transfusion guidelines has a major influence on operating room blood product administration [5, 15]. Patient blood management programs, adoption of guidelines encouraging restrictive transfusion thresholds, and advances in technology and surgical techniques may all contribute to the decrease in overall blood products $[2,10,16]$. Contrary to the trend in other blood products, cryoprecipitate usage has increased nationally [16]. This trend may be secondary to the fluidity in guidelines, discrepancy in practice as compared to national guidelines. The emergence of studies related to cryoprecipitate administration in various fields such as obstetrics, cardiac surgery, and trauma. Have shown that acquired hypofibrinogenemia is associated with worse outcomes and risk of bleeding [11,12, 17, 18].

Strain continues to be placed on blood collection centers as manufacturing costs of safe blood products rises, despite decreasing demand and cost hospitals are willing to pay for blood products $[1,2]$. Appropriate use of cryoprecipitate and reduction of waste can help reduce the chances of rationing care for patients especially in resource constrained hospital systems. There are also financial benefits associated with decreasing misuse and wasting of cryoprecipitate. At our institution, the acquisition cost of cryoprecipitate is $\$ 228$ per unit. That expense doesn't include the cost of storage, preparation, thawing, compatibility testing or transfusion [3, 14]. Cryoprecipitate issued to the operating room but not transfused should be returned to the blood bank at room temperature. Based on our survey responses, if cryoprecipitate was not utilized $41.2 \%$ of responding providers correctly left it in the operating room outside of the blood transport cooler while $50 \%$ put it in the cooler for return to the blood bank resulting in wastage due to improper temperature. Using evidence-based medicine and societal guidelines, we reinforced indications for ordering cryoprecipitate and management of unused cryoprecipitate; specifically, maintaining the unit at room temperature and prompt return to the blood bank at the end of the operation or sooner if not needed. Additionally, a source of waste was the practice of thawing cryoprecipitate just in case the need arises; the reason behind this was the thought that it may take over an hour to deliver the unit to the operating room. This information was elucidated from the survey and clinicians were educated on the average time for cryoprecipitate delivery, which is closer to $30-40 \mathrm{~min}$. This practice was also cautioned against as clinicians were educated that once thawed, the cryoprecipitate unit would have to be transfused within $4 \mathrm{~h}$ otherwise it would be wasted as reallocating the unit was difficult and rarely occurred. Improving upon these practice habits led to a decrease in wasted cryoprecipitate and more appropriate ordering of cryoprecipitate (Fig. 4).

Blood product transfusions are the most common procedures in the United States; however, there are risks associated with receiving blood product transfusions [2, 19]. Specifically with cryoprecipitate transfusions, there is a risk of transmission of blood-borne pathogens and transfusion-related acute

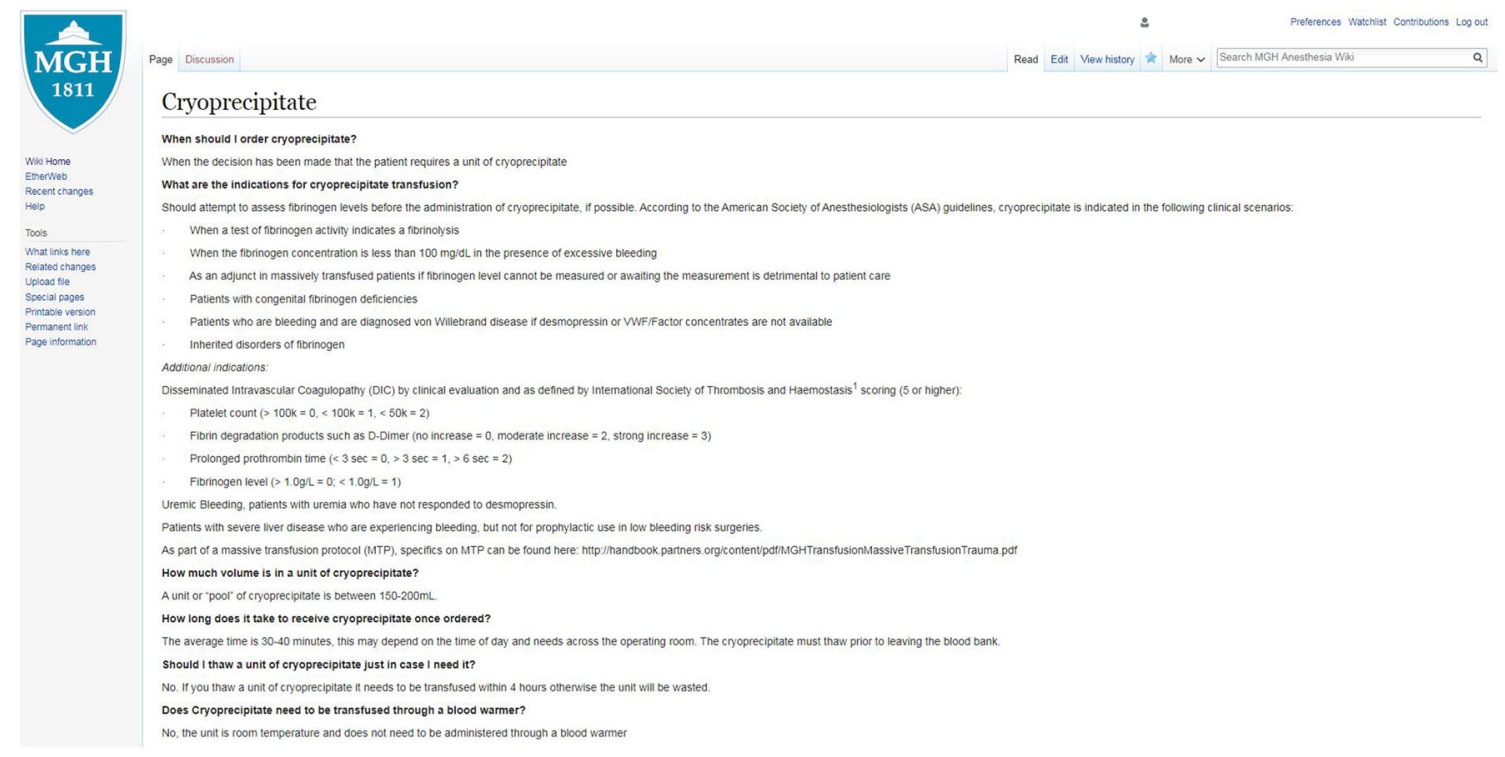

Fig. 3 Picture of Departmental Online Educational Content 
Monthly Cryoprecipitate Usage

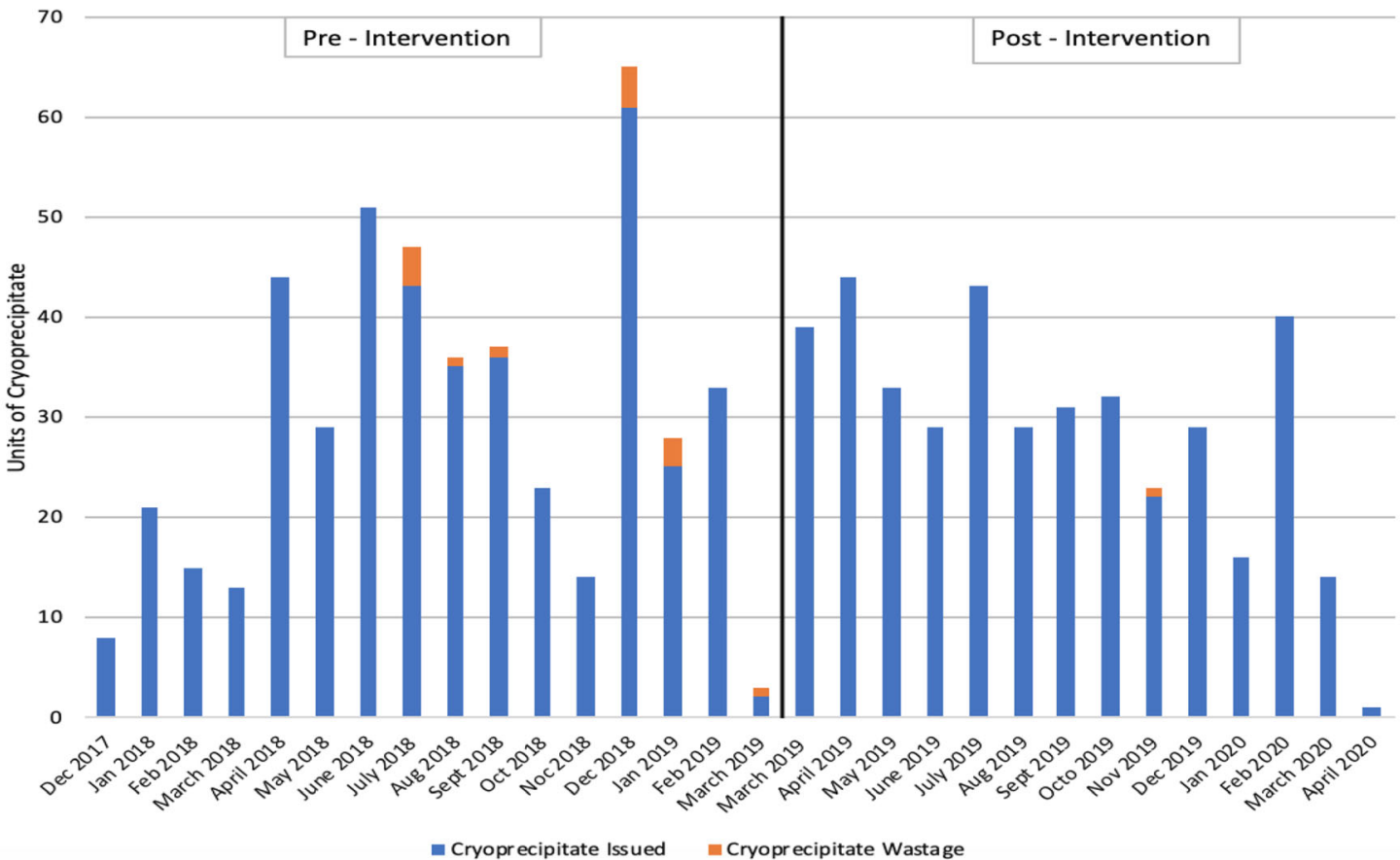

Fig. 4 Stacked column chart demonstrating units of cryoprecipitate issued monthly (blue) compared to units wasted each month (orange). Black line denotes separation between pre and post- intervention data

lung injury (TRALI) $[3,5,6]$. The risk of TRALI was estimated at 1 in 317,000 units of cryoprecipitate in a United Kingdom report from 1996 to 2003 [5]. Although cryoprecipitate can be administered in single units, larger volumes may be required, thus exposing recipients to multiple donors $[3,5,6]$. While there are few reports detailing overall incidence of adverse events, the Quebec hemovigilance system reported 6.57 adverse events per 10,000 units of cryoprecipitate transfused [5].

The transfusion of allogeneic blood products is associated with longer hospital stays and increased health care costs [20]. Blood product transfusions are a crucial therapy in modern medicine, however there are benefits of limiting unnecessary transfusions for both avoidance
Fig. 5 Cryoprecipitate wastage from December 2017-March 2019 (pre-intervention) compared to March 2019-April 2020 (postintervention), columns represent the percentage of cryoprecipitate wastage during each time period

\section{Cumulative Cryoprecipitate Wastage}

3.5

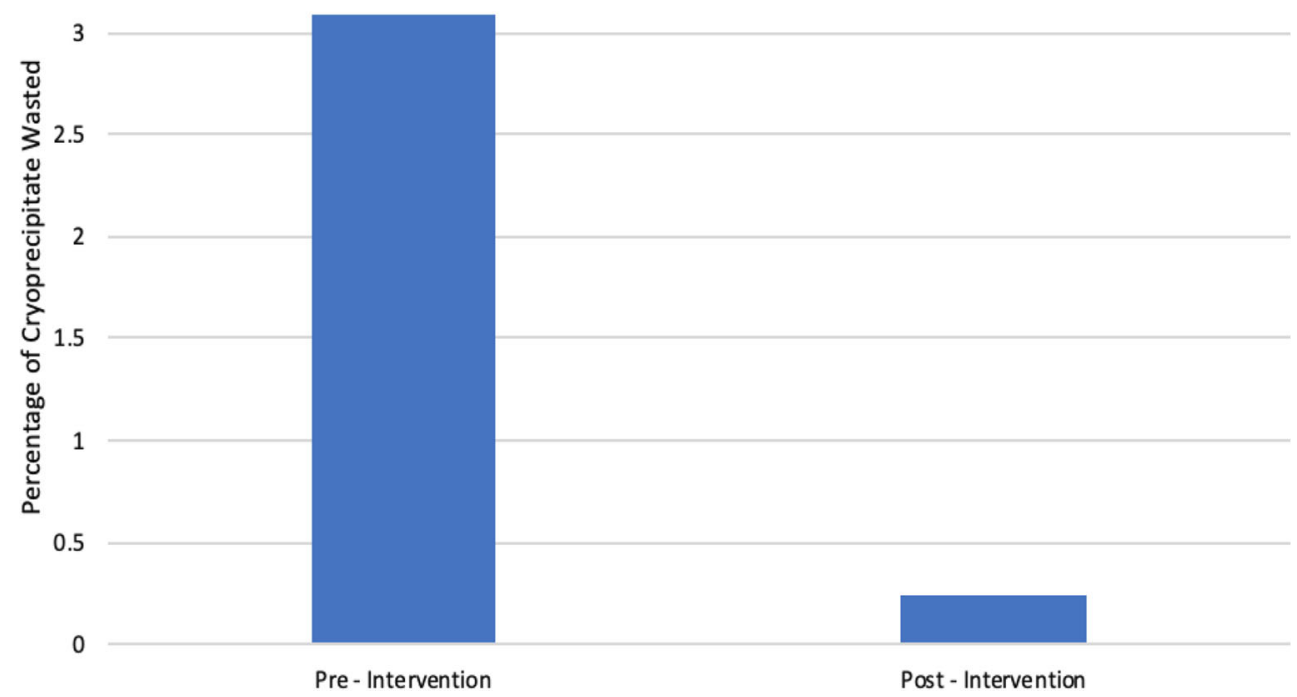


Fig. 6 Comparison of the average cryoprecipitate usage during the pre-intervention versus postintervention periods. Columns represent mean monthly values. $*$ designates significant findings $(P<0.05)$
OR Cryoprecipitate Usage

35

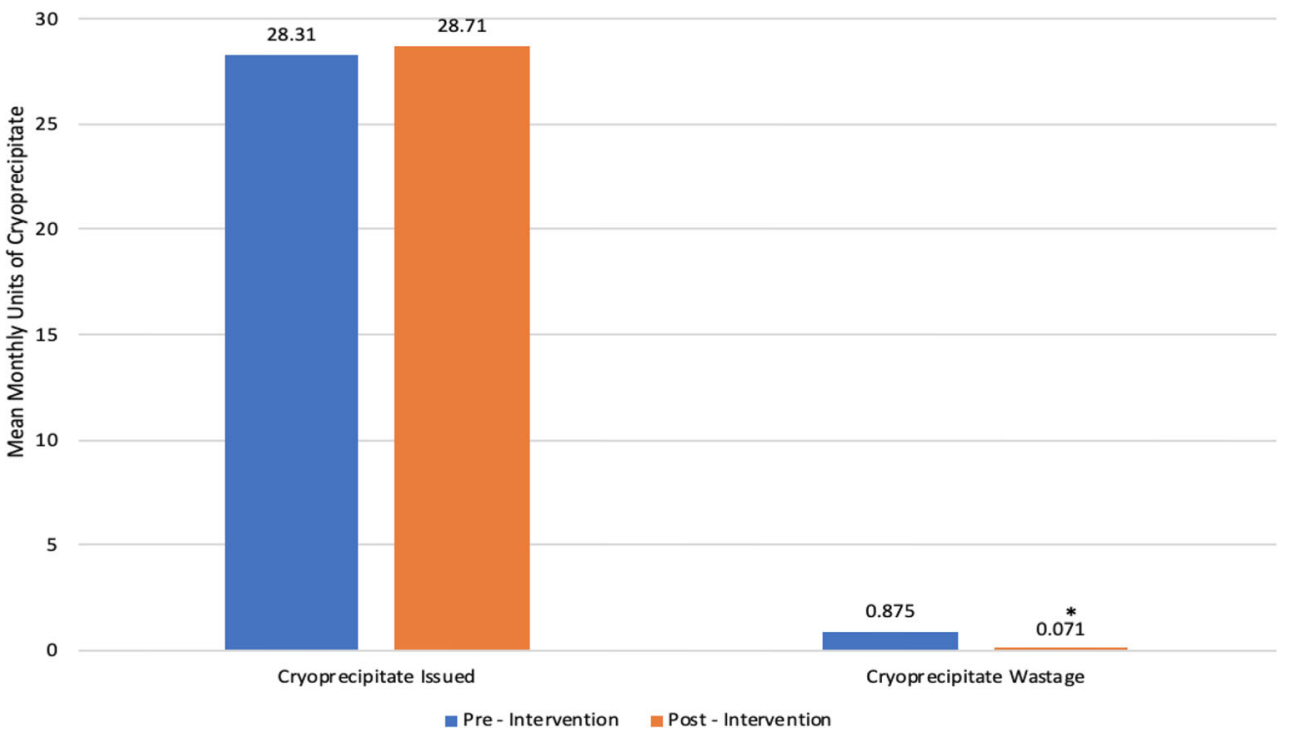

of adverse outcomes and cost reduction. Institution level initiatives involving blood product management and waste reduction can mitigate the above concerns by reducing unnecessary transfusions and provide cost savings.

Limitations of our study include the follow-up post-intervention period being shorter than the pre-intervention period due to reduced operating room volume secondary to the COVID-19 pandemic. Another limitation is these results come from a single institution and do not account for confounding changes in clinical practice. Additionally, we only focused on ordering and returning cryoprecipitate and there are other methods to reduce waste which are outside the scope of our study including the implementation of stricter transfusion thresholds.

\section{Conclusion}

By evaluating ordering practices and utilization of cryoprecipitate at our institution, overall cryoprecipitate wastage was reduced after executing initiatives aimed at addressing improper ordering and handling of cryoprecipitate. These results demonstrate that utilizing educational initiatives for optimization of blood product management can reduce unnecessary ordering, transfusions, and waste; an outcome that could ultimately reduce costs and improve quality of care.

\section{Declarations}

Conflict of interest None relevant to this work.

\section{References}

1. Klein HG, Hrouda JC, Epstein JS. Crisis in the Sustainability of the U.S. Blood System. N Engl J Med. 2017;377(15):1485-1488. doi: https://doi.org/10.1056/NEJMsb1706496

2. Jones JM, Sapiano MRP, Savinkina AA, et al. Slowing decline in blood collection and transfusion in the United States - 2017. Transfusion. 2020;60 Suppl 2(Suppl 2):S1-S9. doi:https://doi.org/ $10.1111 /$ trf. 15604

3. Storch EK, Custer BS, Jacobs MR, Menitove JE, Mintz PD. Review of current transfusion therapy and blood banking practices. Blood Rev. 2019;38:100593. doi:https://doi.org/10.1016/j.blre. 2019.100593

4. Chung KW, Basavaraju SV, Mu Y, et al. Declining blood collection and utilization in the United States. Transfusion

5. Nascimento B, Goodnough LT, Levy JH. Cryoprecipitate therapy. Br J Anaesth. 2014;113(6):922-934. doi:https://doi.org/10.1093/ bja/aeu158

6. Yang L, Stanworth S, Baglin T. Cryoprecipitate: an outmoded treatment?. Transfus Med. 2012;22(5):315-320. doi:https://doi.org/10. 1111/j.1365-3148.2012.01181.x

7. Ciavarella D, Reed RL, Counts RB, Baron L, Pavlin E, Heimbach $\mathrm{DM}$, et al. Clotting factor levels and the risk of diffuse microvascular bleeding in the massively transfused patient. Br J Haematol 1987;67(3):365-8.

8. American Society of Anesthesiologists Task Force on Perioperative Blood Management. Practice guidelines for perioperative blood management: an updated report by the American Society of Anesthesiologists Task Force on Perioperative Blood Management. Anesthesiology. 2015;122: 241-275

9. Fries D, Martini WZ. Role of fibrinogen in trauma-induced coagulopathy. Br J Anaesth 2010; 105: 116-21

10. Levy JH, Szlam F, Tanaka KA, Sniecienski RM. Fibrinogen And Hemostasis:a primary hemostatic target for the management of acquired bleeding. Anesth Analg 2012; 114: 261-74

11. Anderson MA, Glazebrook B, Cutts B, Stevenson L, Bielby L, Borosak M. When do we transfuse cryoprecipitate?. Intern Med J. 2013;43(8):896-902. doi:https://doi.org/10.1111/imj.12104 
12. McQuilten ZK, Bailey M, Cameron PA, Stanworth SJ, Venardos $\mathrm{K}$, Wood EM, et al. Fibrinogen concentration and use of fibrinogen supplementation with cryoprecipitate in patients with critical bleeding receiving massive transfusion: a bi-national cohort study. Br J Haematol 2017;179(1):131-41.

13. Ranucci M, Solomon C. Supplementation of fibrinogen in acquired bleeding disorders: experience, evidence, guidelines, and licences. Br J Anaesth 2012;109(2):135-7

14. Toner RW, Pizzi L, Leas B, Ballas SK, Quigley A, Goldfarb NI. Costs to hospitals of acquiring and processing blood in the US: a survey of hospital-based blood banks and transfusion services. Appl Health Econ Health Policy. 2011;9:29-37

15. Whitaker BI, Kamani N. Surveys of blood collection and utilization. Transfusion 2018;58(2):541-2.

16. Ellingson KD, Sapiano MRP, Haass KA, et al. Continued decline in blood collection and transfusion in the United States-2015. Transfusion 2017;57(Suppl 2):1588-98

17. Gillissen A, van den Akker T, Caram-Deelder C, Henriquez D, Bloemenkamp KWM, de Maat MPM, et al. Coagulation parameters during the course of severe postpartum hemorrhage: a nationwide retrospective cohort study. Blood Adv 2018;2(19): 2433-42.

18. McQuilten ZK, Wood EM, Bailey M, Cameron PA, Cooper DJ. Fibrinogen is an independent predictor of mortality in major trauma patients: a five-year statewide cohort study. Injury 2017;48(5): 1074-81.

19. Pfuntner A, Wier LM, Stocks C. Most frequent procedures performed in U.S. hospitals, 2010. HCUP statistical brief \#149. Rockville (MD): Agency for Healthcare Research and Quality; 2013.

20. Popovsky MA, Audet AM, Andrzejewski C Jr. Transfusion associated circulatory overload in orthopedic surgery patients: a multiinstitutional study. Immunohematology 1996; 12: 87-9

Publisher's Note Springer Nature remains neutral with regard to jurisdictional claims in published maps and institutional affiliations. 